\title{
Clinical update on the use of ospemifene in the treatment of severe symptomatic vulvar and vaginal atrophy
}

This article was published in the following Dove Press journal:

International Journal of Women's Health

26 October 2016

Number of times this article has been viewed

\author{
Santiago Palacios' \\ María Jesús Cancelo² \\ 'Palacios Institute of Women's Health, \\ Madrid, Spain; ${ }^{2}$ Gynecology and \\ Obstetrics Department, Guadalajara \\ University Hospital, University of \\ Alcalá, Spain
}

\begin{abstract}
The physiological decrease in vaginal estrogens is accountable for the emergence of vulvar and vaginal atrophy (VVA) and its related symptoms such as vaginal dryness, dyspareunia, vaginal and/or vulvar irritation or itching, and dysuria. The repercussion of these symptoms on quality of life often makes it necessary to initiate treatment. Up until now, the treatments available included vaginal moisturizers and lubricants, local estrogens, and hormonal therapy. However, therapeutic options have now been increased with the approval of $60 \mathrm{mg}$ ospemifene, the first nonhormonal oral treatment with an agonist effect on the vaginal epithelium and an endometrial and breast safety profile which makes it unique. This is the first selective estrogen receptor modulator indicated in women with moderate-to-severe vaginal atrophy not eligible for local estrogen treatment. Considering that "local estrogen noneligible women" are those in whom such treatment cannot be administered either because it is contraindicated or due to skill issues, who are averse to the mode and convenience of vaginal products' administration or to their use on account of potential systemic absorption, or those who demonstrate dissatisfaction in terms of efficacy and safety, it is clear that there is a significant unmet medical need in VVA management. In fact, a great number of women show lack of adherence, dropping out of at least one VVA treatment, including nonhormonal moisturizers and lubricants, which they consider to be ineffective and uncomfortable. If they could choose, many of them may opt for oral treatment. In Phase III studies, ospemifene demonstrated efficacy in vaginal dryness and dyspareunia, regenerating vaginal cells, improving lubrication, and reducing pain during sexual intercourse. Symptoms improved in the first 4 weeks and endured for up to 1 year. Additionally, it demonstrated a good endometrial, cardiovascular system, and breast safety profile.
\end{abstract}

Keywords: ospemifene, vulvar and vaginal atrophy, dyspareunia, therapeutic options

\section{Introduction}

The physiological decrease of vaginal estrogens modifies the characteristics of the vaginal epithelium, which becomes squamous and stratified, whereas it was wet and wrinkled before menopause. ${ }^{1}$ Under hypoestrogenism conditions, the vaginal epithelium becomes thinner, vaginal elasticity decreases, connective tissue increases, and vaginal blood flow is reduced. The decrease in surface epithelial cells below 5\% means lower cell exfoliation, lower glycogen release, and lower hydrolysis to glucose, which causes reduced lactobacillus-induced transformation to lactic acid and increased vaginal $\mathrm{pH}$ over 4.5. ${ }^{1}$ The alkaline environment encourages a change in bacterial microbiota, which implies a risk of dysbiosis. ${ }^{1}$ In addition, vaginal stenosis and risk of damage as a result of vaginal elasticity loss, as well as lubrication reduction owing to lower blood inflow, can bring about some of the typical symptoms of vulvar and vaginal atrophy (VVA),
Correspondence: Santiago Palacios Palacios Institute of Women's Health, Calle de Antonio Acuña, 9, 28009 Madrid, Spain

Tel +349l 5780517

Email spalacios@institutopalacios.com 
such as vaginal dryness, dyspareunia, vaginal and/or vulvar irritation or itching, dysuria, and vaginal bleeding associated with sexual intercourse. ${ }^{1}$

A new terminology has been proposed to refer to vulvovaginal atrophy or atrophic vaginitis: genitourinary syndrome of menopause. It is considered that the two terms that have been used so far, vulvovaginal atrophy and atrophic vaginitis, do not include the variety of signs and symptoms that occur during this time; especially, they do not include the ones related to urinary tract symptoms (dysuria, nocturia, etc). It has also been seen that not all people are comfortable talking about the vulva and/or vagina and the term atrophy may have a derogatory connotation. ${ }^{2}$

Genitourinary Syndrome of Menopause (GSM) or VVA diagnosis is clinical, and apart from symptoms, it is supported by the visual examination of clinical signs such as pallor, redness, petechiae, dryness, and friability of the genital mucosa. Other parameters supporting diagnosis and helping differential diagnosis to be carried out include vaginal $\mathrm{pH}$ and vaginal maturation index (MI). Given that the epithelial covering of the vaginal wall is extremely estrogen-sensitive, the cell proportion in the superficial, intermediate/superficial spinous, and spinous/parabasal cell strata is suggestive of estrogen stimulation and is used to assess the estrogenization degree of the vaginal epithelium. ${ }^{3}$ Vaginal MI is reduced in postmenopausal women owing to the decrease in superficial cells below 5\%. Nevertheless, these laboratory data or clinical signs at visual examination are not necessarily correlated with VVA symptoms. ${ }^{3}$ The same applies to the clinical signs of estrogen deficiency in the vaginal tissue, which are not always associated with severe or persistent vaginal symptoms in all women either. ${ }^{4}$ A clear example of this is Gass et al's work, ${ }^{5}$ where only $10 \%$ of the patient population with clear signs of VVA at physical exploration reported moderate-to-severe vaginal dryness. This suggests that there are certain contextual and behavioral factors which can have a more significant impact on women's subjective experience about their own symptoms than purely physical signs of atrophy or inflammation. ${ }^{6}$

The prevalence and impact of vulvar and vaginal symptoms on postmenopausal women's quality of life often makes it necessary to initiate treatment. This review analyzes the clinical data available with ospemifene, the first selective estrogen receptor modulator (SERM) indicated in women with VVA. For that purpose, a limited literature search was conducted on key resources, including PubMed, the Cochrane Library, Corporate Receivables Inc - Collection Agency, and major international health technology agencies. Filters were applied to specific questions related to ospemifene, VVA, and dyspareunia. The search was also limited to English language.

\section{Treatment options Hormone therapy $(\mathrm{HT})$}

For decades, HT with estrogens either in monotherapy or combined with progestogens has been the treatment of choice for menopausal symptoms' relief and osteoporosis prevention. ${ }^{7}$ $\mathrm{HERS}^{8}$ and $\mathrm{WHI}^{9}$ study findings changed the previous perception of HT's risk-benefit as they showed an increased risk of cardiovascular disease, ${ }^{8,9}$ thromboembolism, and invasive breast cancer associated with estrogens. Since then, HT is no longer recommended for chronic disease prevention, ${ }^{7,10}$ not even in breast cancer patients. ${ }^{7,9,11,12}$ However, there is evidence supporting a different approach to HT management of menopausal symptoms by employing the lowest possible dose and individualizing treatment based on patient age and symptom severity. Currently, systemic HT is not indicated in patients with VVA as their only menopausal symptom. ${ }^{13}$

\section{Local estrogen therapy}

For VVA cases with no menopausal symptoms other than hot flashes, local estrogen therapy is recommended. ${ }^{14}$ Apart from preventing systemic hormone therapy side-effects, it is probably more effective in relieving vulvar and vaginal symptoms and improving cytological results, ${ }^{15}$ whether used in creams, rings, ovules, or tablets. ${ }^{16}$ Local estrogens are highly effective in reverting physiological changes associated with VVA; they foster vaginal cell growth and maturation, favor lactobacillus recolonization, improve blood flow, lower $\mathrm{pH}$, increase vaginal epithelium thickness and elasticity, and enhance sexual function. ${ }^{15,17,18}$ There is evidence that the effects of these intravaginal preparations are not limited to the vagina ${ }^{13,19}$ and that there is a risk of systemic absorption due to the vaginal atrophic state, especially during the first days of treatment. ${ }^{13,18,19}$ However, an increase in plasma estrogen levels with ultralow doses of local estrogen therapy has not been demonstrated $;{ }^{20}$ therefore, it is not necessary to add a progestogen to establish controls to asses endometrial stimulus. ${ }^{21,22}$ As the epithelium matures as a result of treatment, absorption decreases and small estrogen doses are required to prevent recurrent atrophy. ${ }^{23}$

To meet treatment goals, it is recommended to apply the lowest local estrogen dose possible over the shortest time possible. ${ }^{18,21,22}$ A low dose below $50 \mathrm{mcg}$ per application is expected $^{21,22}$ to induce as few systemic effects as possible and maintain plasma estrogen levels within the normal 
postmenopausal range. ${ }^{21,22}$ Concerns about a potential systemic absorption of intravaginal estrogens and their stimulating effects on the breast and the endometrium, among other adverse events (AEs), can prevent long-time use. ${ }^{19,24}$ Indeed, 12\%-38\% of local estrogen-treated REVIVE survey respondents ${ }^{21}$ and $33 \%$ of VIVA survey respondents ${ }^{25}$ admitted that they were concerned about hormone exposure, and $30 \%$ of VIVA respondents ${ }^{25}$ were reluctant to use local estrogen therapy. As a matter of fact, safety was the element women were more dissatisfied with $(40 \%)$, followed by efficacy $(25 \%)$.

\section{Vaginal moisturizers and lubricants}

Local estrogen administration may be contraindicated in women with undiagnosed uterine/vaginal bleeding, or with a previous history of hormone-dependent tumors such as breast, ovary or endometrial cancer and cervical adenocarcinoma. ${ }^{21,26}$ It can also prove uncomfortable in women with skill problems (for instance, arthritis, obesity or stroke). In such cases, and also in women who wish to avoid hormone treatment, guidelines recommend vaginal moisturizers or lubricants as first-line treatment. ${ }^{13,16,21,27}$ In addition, the use of vaginal moisturizers or lubricants to relieve dryness symptoms in women not responding to estrogens should also be considered. ${ }^{13}$ These products do not treat the underlying condition, ${ }^{13}$ but do provide temporary relief of vaginal atrophy symptoms, ${ }^{28}$ such as dryness and pain during sexual intercourse ${ }^{13}$ with lubricant use. Moisturizers' benefits may last longer if used continuously, ${ }^{13,29}$ but their efficacy in vaginal symptoms is lower as compared to local estrogens, ${ }^{28}$ and less than $50 \%$ of postmenopausal women in Europe are satisfied with their moisturizer or lubricant. Use is sometimes associated with vaginal irritation, ${ }^{13}$ contact dermatitis, and allergy. ${ }^{30}$

Other natural products for vaginal health include phytoestrogens, which are nonsteroidal vegetal compounds with estrogen receptor (ER) binding capacity. Some studies suggest beneficial effects on the urogenital sphere ${ }^{31,32}$ as they improve symptoms of dyspareunia, vaginal dryness, and maturation value. ${ }^{33}$ Given their estrogen agonist effects and the lack of safety data in women with estrogen-sensitive tumors, phytoestrogens should be recommended cautiously. ${ }^{13}$

\section{SERMs}

SERMs are a heterogeneous group made up of nonsteroidal compounds mostly which act as estrogen receptor (ER) ligands but were developed as targeted therapies. ${ }^{34}$ In other words, contrary to estrogens, which usually have an agonist activity, SERMs exert a mixed agonist or antagonist action depending on the target tissue. ${ }^{35-38}$ This represents a pharmacological advantage as it prevents AEs in nontarget tissues. ${ }^{39}$ SERMs' tissue-specific activity is partly determined by a differential and specific affinity for ER $\alpha$ or $\beta$ subtypes, with a differential tissue expression pattern each, and by the formation of ligand-receptor complexes with differential effects on gene regulation depending on ER $\alpha$ or ER $\beta$ binding. ${ }^{40,41}$

The importance of ER-mediated signaling in normal tissues shows the therapeutic potential of selective ER binding in the management of postmenopausal disorders associated with estrogen deficiency, such as osteoporosis. ${ }^{42}$ This is one of the main clinical applications of SERMs in postmenopausal women, along with the maintenance of a beneficial serum lipid profile ${ }^{39}$ and oncologic treatment. ${ }^{43}$ However, potentially severe adverse reactions (ARs) such as thromboembolic events and uterine cancer complicate their long-term use required both for osteoporosis prevention and breast cancer prevention and treatment. ${ }^{39,44}$ The idea of an ideal SERM, would be one that acts as an agonist agent in the bone, brain, and cardiovascular system, and as a neutral or antagonist agent in the endometrium and breast. ${ }^{42}$ But for now, each SERM must be considered individually and depending on their specific therapeutic indication. An example of that is tamoxifen, the first SERM used in clinical practice and the most effective agent in ER-positive breast cancer treatment owing to its breast antiestrogenic activity. Nevertheless, its agonist effect in the uterus and its endometrial hyperplasia-associated risk limits its use to breast cancer postmenopausal population, ${ }^{42}$ thus being ruled out as a potential treatment for postmenopausal osteoporosis, since risks are more important than bone protection benefits. ${ }^{44}$ Raloxifene is a SERM with antagonist estrogen effect in the breast and a neutral one in the uterus which has demonstrated endometrial safety, efficacy in bone fracture and bone loss prevention, and an added benefit in breast cancer prevention, ${ }^{44}$ but which induces or exacerbates hot flashes. ${ }^{45}$ Other SERMs currently used in clinical practice include clomiphene, approved in the US for ovulatory dysfunction treatment in women desiring to get pregnant, ${ }^{40}$ toremifene in postmenopausal women with metastatic hormone dependent breast cancer, ${ }^{46}$ and bazedoxifene for osteoporosis treatment in women with increased fracture risk. ${ }^{47}$

Selectively targeted SERMs against VVA's underlying physiopathology could be an alternative to local estrogen or systemic hormone therapy in the management of VVA symptoms in women with a previous history of breast cancer or currently with breast cancer, or simply in women who prefer 
to avoid such treatments. However, tamoxifen, raloxifene, and bazedoxifene as monotherapy are not currently indicated in VVA treatment. ${ }^{48-52}$ Briefly, tamoxifen has demonstrated an agonist and antagonist activity in the vaginal epithelium, ${ }^{53}$ as well as gynecological adverse effects such as dyspareunia and vaginal dryness. ${ }^{50,52}$ The evidence available for raloxifene does not show any effect on postmenopausal monotherapy-treated women's urogenital epithelium, ${ }^{48,50}$ or in combination with vaginal estrogens as it provides no additional benefit in relieving genitourinary atrophy signs and symptoms. ${ }^{53-55}$ Data on bazedoxifene, although limited, conclude that it could only be a safe and effective option in combination with conjugated estrogens as it improves VVA measures ${ }^{56,57}$ and reduces the incidence of dyspareunia, among other moderate-to-severe VVA symptoms. ${ }^{58}$

Among marketed SERMs, only ospemifene (a toremifene derivative) has an estrogenic agonist activity on the vaginal epithelium already noted in the first nonclinical studies ${ }^{59}$ and Phase I, II and III studies. ${ }^{60}$ This singular tissue selectiveness seems to be molecular structure-related ${ }^{61}$ It is the first nonhormonal selective modulator with multiple tissue-specific actions and, contrary to other SERMs, with an antagonist function in endometrial and breast tissue..$^{59,62,63}$

\section{Ospemifene}

\section{Clinical development}

Ospemifene's clinical development included 21 Phase I studies and nine Phase II/III studies, as well as a population pharmacokinetics analysis and 12 nonclinical in vitro studies with human samples. ${ }^{64}$ In the Phase II/III studies, a total of 1,892 postmenopausal women, with and without uterus, were given at least one dose of ospemifene, approximately $80 \%$ of whom received a dose $\geq 60 \mathrm{mg}$ daily. ${ }^{64}$ Seventy-two percent of patients were treated for at least 12 weeks, $35 \%$ for at least 6 months, and $22 \%$ for at least 1 year, with a maximum exposure of 89 weeks. $^{64}$

\section{Phase III studies}

Aside from the 12-week Phase II, randomized, double-blind, placebo-controlled study (Study 717) ${ }^{64}$ to assess ospemifene's minimum effective dose $(5 \mathrm{mg} /$ $15 \mathrm{mg} / 30 \mathrm{mg}$ ), clinical efficacy in symptomatic VVA treatment in postmenopausal women was primarily determined in three Phase III multi-center, randomized, double-blind, placebo-controlled studies: two 12-week reference studies

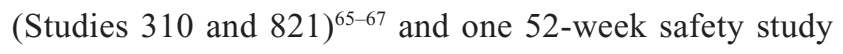
(Study 718) ${ }^{68}$ (Figure 1). Once the ospemifene initial reference study's (Study 310) ${ }^{65}$ double-blind phase was

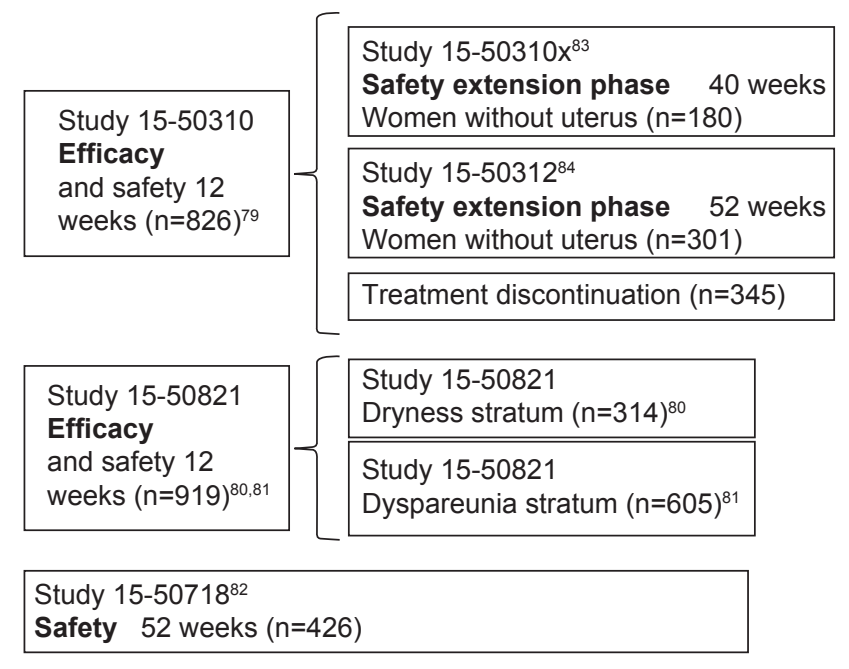

Figure I Ospemifene $60 \mathrm{mg}$ Phase III program.

completed, patients with an intact uterus could voluntarily choose to undergo an open-label extension phase with $60 \mathrm{mg}$ ospemifene for up to 40 weeks (Study 310x), ${ }^{69}$ and hysterectomized patients could choose to undergo an openlabel extension phase for up to 52 weeks (Study 312) 70 $^{7}$ order to assess long-term safety. This led to the approval of oral ospemifene $(60 \mathrm{mg} /$ day $)$ as the first nonhormonal SERM for moderate-to-severe dyspareunia treatment in VVA postmenopausal women. ${ }^{71}$

Both 12-week reference studies included postmenopausal women aged 40-80 years with the following VVA criteria: $\leq 5 \%$ of superficial cells at vaginal smear, vaginal $\mathrm{pH}>5.0$, at least one moderate or severe VVA symptom (Study 310$),{ }^{65}$ or moderate-to-severe vaginal dryness or sexual activity associated dyspareunia (Study 821). ${ }^{66,67}$ In the 52-week safety study (Study 718), ${ }^{68}$ patients had to have an intact uterus, although they were not selected based on their VVA symptoms.

Primary endpoints of both 12-week reference studies included changes in parabasal and superficial cell percentage from baseline in the vaginal smear's MI, changes in vaginal $\mathrm{pH}$, and changes in severity of the most troublesome symptom (MTS), ranked on a 4-point scale (0: none; 1: mild; 2: moderate; 3 : severe). Although safety was the 52 -week Study 718 's primary goal, ${ }^{68}$ data on $60 \mathrm{mg}$ ospemifene efficacy at 12 weeks in objective measurements, superficial and parabasal cell percentage, and vaginal $\mathrm{pH}$ were also collected.

Secondary endpoints differed among studies. They included changes in VVA physiological markers at

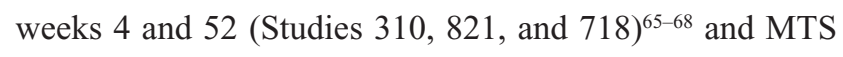
severity at week 4 (Studies 310 and 821 ), ${ }^{65-67}$ percentage of 
responding patients (Study 821), ${ }^{66,67}$ as well as lubricant use (Studies 310, 821) ${ }^{65-67}$ and sexual function at weeks 4 and 12 (Study 821). ${ }^{66,67}$ Patients with a MI 10-point increase, a vaginal $\mathrm{pH} 0.5$ decrease, and a vaginal dryness severity 1 -point reduction were considered as responding. Both in the 52-week safety study and in the 40- and 52-week extension phases of ospemifene's initial reference study, changes observed at vaginal visual exploration regarding clinical signs of petechiae, pallor, friability, dryness, and mucosal redness with their severity ranked on a 4-point scale ( 0 : none; 1 : mild; 2: moderate; 3 : severe) - were analyzed.

\section{Therapeutic efficacy}

In the three Phase III studies, age, race, and body mass index demographic characteristics were comparable between both treatment arms, and discontinuation rates were low, with more than $80 \%$ of patients completing the $60 \mathrm{mg}$ ospemifene 12- and 52-week treatment.

\section{Effects on vaginal epithelium maturation and vaginal $\mathrm{pH}$} After 4 weeks (Study 310), ${ }^{65}$ and 12 weeks (Studies 310 and 821), ${ }^{65-67} 60 \mathrm{mg}$ ospemifene reduced the parabasal cell percentage $(-30.1,-31.7,-40.3)$ and vaginal $\mathrm{pH}$ $(-1.01,-0.95,-0.94)$ significantly, and increased superficial cell percentage $(10.8,7.0,7.0)$ as compared to placebo $(P<0.001)$. This significant changes were consistent among studies endured for 52 weeks in the long-term Study (718), ${ }^{68}$ with a reduction of 40 in the percentage of parabasal cells and -1.22 of vaginal $\mathrm{pH}$, and increased of 5 of the percentage of superficial cells (Study 718). ${ }^{68}$

\section{Effects on vaginal dryness and dyspareunia}

In women reporting vaginal dryness and dyspareunia as the MTS in Study 310, ${ }^{65} 60 \mathrm{mg}$ ospemifene reduced both symptoms' severity significantly after 12 weeks of treatment as compared to placebo. Seventy-five percent of patients with vaginal dryness experienced improvement ( $\geq 1$-point decrease in severity score), and 66\%, relief (with no symptoms or mild symptoms). In the patient cohort who identified vaginal dryness as the MTS in Study 821, $60 \mathrm{mg}$ ospemifene reduced the degree of severity reported from baseline significantly $(-1.3)$ as compared to placebo $(-1.1)$ $(P=0.080),{ }^{66}$ and $46.3 \%$ of patients experienced substantial improvement (2-point or 3-point decrease in severity score) as compared to $34.4 \%$ treated with placebo $(P<0.05) .{ }^{72}$ In the patient cohort who identified dyspareunia as the MTS, $60 \mathrm{mg}$ ospemifene also reduced the degree of severity significantly $(-1.5)$ as compared to placebo $(-1.2)(P<0.0001)$, and increased the percentage of patients who experienced improvement ( $80 \%$ vs $64 \%$ with placebo; $P<0.001$ ), substantial improvement (53\% vs 39\% with placebo; $P<0.0001$ ), or relief (63\% vs $42.5 \%$ with placebo; $P<0.0001) .{ }^{67}$ Responding patient percentage was significantly higher in the $60 \mathrm{mg}$ ospemifene arm as compared to the placebo arm (Study 310 [33.7\% vs 3.4\%; $P<0.001]$, Study 821-dryness stratum [33.8\% vs 7.1\%; $P<0.0001]$, Study 821 -dyspareunia stratum $[42.9 \%$ vs $4.6 \% ; P<0.0001])$.

\section{Effects on VVA clinical signs}

Ospemifene improved VVA clinical signs substantially both in hysterectomized women ${ }^{70}$ and in women with intact uterus. ${ }^{69}$ Vaginal visual examination demonstrated actual improvements in vaginal dryness, redness, petechiae, pallor, and mucosal friability at weeks $12^{73}$ and $26,{ }^{69,70,73}$ enduring for the whole 52-week $60 \mathrm{mg}$ ospemifene treatment period as compared to placebo, ${ }^{68-70}$ with the majority of patients having no or mild VVA clinical signs at week $52 .{ }^{68,70}$

\section{Effects on lubricant use and female sexual function}

Ospemifene $60 \mathrm{mg}$ clinical benefit was also demonstrated in lubricant use and sexual activity. In Studies 310 and 821, ${ }^{65-67}$ there was a trend toward lower lubricant use in the $60 \mathrm{mg}$ ospemifene group vs placebo, with a slight reduction in the percentage of women reporting use following 3 weeks of treatment, and throughout the whole 12 weeks of the studies. Ospemifene $60 \mathrm{mg}$ was also significantly more effective than placebo in terms of sexual activity improvement, measured through total Female Sexual Function Index score, in weeks 4 (5.29 vs 3.70; $P<0.001$ ) and 12 (6.69 vs $4.14 ; P<0.001$ ). This improvement, which began to show up in the first 4 weeks of treatment with significant pain reduction during sexual intercourse $(P<0.05$ vs placebo) and higher lubrication $(P<0.001$ vs placebo), endured throughout the whole 12 weeks in the Female Sexual Function Index domains: arousal $(P<0.05$ vs placebo), desire $(P<0.001$ vs placebo), orgasm $(P<0.001$ vs placebo), lubrication $(P<0.0001$ vs placebo), satisfaction $(P<0.05$ vs placebo $)$, and pain $(P<0.0001$ vs placebo $){ }^{74}$ These results were consistent both in hysterectomized women and women with intact uterus, and also in women with dyspareunia and vaginal dryness as the MTS. $^{74}$

\section{Clinical application of efficacy data}

Symptom improvement is a primary efficacy measurement based on ospemifene-treated women's mean change in MTS severity, ranked on a 4-point scale ( 0 : no symptom; 1: mild; 2: moderate; 3: severe). 
All ospemifene results described previously demonstrate the treatment's statistical significance. However, the clinical interpretation of data is essential not only for the physician, who may not be familiar with the use of numerical severity measurements within their clinical practice, but also for the patients to understand what they can actually expect from ospemifene treatment.

Nappi et $\mathrm{al}^{72}$ analyzed the clinical relevance of Study 310 and Study 821 results, ${ }^{65-67}$ based on two concepts previously described $^{75}$ (improvement and relief), and developing a new concept of "substantial improvement". ${ }^{65}$ This way, authors demonstrated that, after 12-week ospemifene treatment, up to $75 \%$ of women experienced at least a 1-point improvement in their vaginal dryness symptoms, and up to $80 \%$ in their dyspareunia symptoms. ${ }^{66,67}$ What is more, up to $66 \%$ and $63 \%$ of women claimed to have no or mild symptoms of vaginal dryness $^{65}$ and dyspareunia, ${ }^{66,67}$ respectively. In addition, up to $46 \%$ and $53 \%$ of women experienced at least a 2-point improvement in their symptoms of vaginal dryness and dyspareunia. ${ }^{66,67}$ The last numbers may be the most relevant ones, since, according to the authors, a 1-point symptom severity improvement can occur spontaneously even without any therapeutic intervention whatsoever. This was reflected in at least $14.9 \%$ of patients from Studies 310 and 821 , who experienced subjective improvement in their symptoms over the 6 months without treatment since the selection and randomization process, vs $0.2 \%$ who experienced substantial improvement. Given that substantial improvement is much less likely to occur without treatment, the difference observed with ospemifene vs placebo reflects the true treatment effect. ${ }^{76}$ According to the authors, these clinically relevant changes in vaginal dryness and dyspareunia, which also showed up following 4 weeks of treatment, along with changes in MI, suggest that ospemifene is not only effective in relieving VVA symptoms, but also in treating the cause of vaginal atrophy.

\section{Safety and tolerability}

The $60 \mathrm{mg}$ ospemifene safety profile was established in more than 2,000 women participating in the randomized, double-blind, placebo-controlled Phase II/III studies ${ }^{65-69}$ and is based on more than 540 patient-years of treatment exposure consistent with long-term use requirements from The International Conference on Harmonisation of Technical Requirements for Registration of Pharmaceuticals for Human Use/European Union guides. The number of $60 \mathrm{mg}$ ospemifene-treated patients was 1.3 times the number of placebo-treated patients, and the number of patient-years with $60 \mathrm{mg}$ ospemifene treatment was 2.0 times the number of patient-years with placebo treatment. ${ }^{65-69}$

Daily $60 \mathrm{mg}$ /day ospemifene treatment was generally well tolerated both in the short and long term, with an $\mathrm{AE}$ global incidence of $67.6 \%$, mostly mild to moderate. Severe $\mathrm{AE}$ incidence was $2.6 \%$ for $60 \mathrm{mg}$ ospemifene and $1.8 \%$ for placebo, with a low rate of severe treatment-related AEs in both groups ( $0.6 \%$ and $0.1 \%$, respectively). ${ }^{65-69}$

Global discontinuation rate was $14.6 \%$ for $60 \mathrm{mg}$ ospemifene and $12.8 \%$ for placebo, and discontinuation rate due to AEs was $7.6 \%$ and $3.7 \%$, respectively. With an AR incidence of $30.4 \%$, hot flashes were the most frequent $60 \mathrm{mg}$ ospemifene-related AEs, with at least one episode in $7.5 \%$ of patients vs $2.6 \%$ in the placebo group. This frequency was lower than that reported for tamoxifen, raloxifene, toremifene, and bazedoxifene. ${ }^{65-69}$ Other common AEs occurring in $\geq 1 / 100$ of patients in any group are presented in Table 1 .

\section{Endometrial and breast safety}

Ospemifene $60 \mathrm{mg}$ endometrial safety in terms of hyperplasia and carcinoma risk was established in the clinical development program as no clinically relevant effect was noted in endometrial histology following 1 year of treatment, except for a single case of simple hyperplasia without

Table I Summary of $60 \mathrm{mg}$ ospemifene-related adverse events

\begin{tabular}{|c|c|c|c|}
\hline \multirow[t]{3}{*}{ Adverse events } & \multicolumn{3}{|c|}{ Number (\%) of patients } \\
\hline & \multicolumn{3}{|c|}{ Phase II/III placebo-controlled studies ${ }^{78-84}$} \\
\hline & Placebo $(n=958)$ & $60 \mathrm{mg}$ ospemifene $(1,242)$ & Frequency \\
\hline \multicolumn{4}{|l|}{ Infections and infestations } \\
\hline Vulvovaginal candidiasis/mycotic infections & $10(0.8)$ & 91 (7.3) & Frequent $(\geq \mathrm{I} / \mathrm{I} 00$ to $<\mathrm{I} / \mathrm{I} 0)$ \\
\hline \multicolumn{4}{|l|}{ Vasomotor disorders } \\
\hline Hot flashes & $25(2.6)$ & $93(7.5)$ & Frequent $(\geq \mathrm{I} / 100$ to $<\mathrm{I} / \mathrm{I} 0)$ \\
\hline \multicolumn{4}{|l|}{ Musculoskeletal and connective tissue disorders } \\
\hline Muscle spasms & $9(0.9)$ & $40(3.2)$ & Frequent $(\geq \mathrm{I} / 100$ to $<\mathrm{I} / \mathrm{I} 0)$ \\
\hline \multicolumn{4}{|l|}{ Reproductive system and breast disorders } \\
\hline Vaginal and genital flow & $4(0.4)$ & $63(5.1)$ & Frequent $(\geq \mathrm{I} / 100$ to $<\mathrm{I} / 10)$ \\
\hline Endometrial hypertrophy & $0(0.0)$ & $9(0.7)$ & Nonfrequent $(\geq \mathrm{I} / \mathrm{I}, 000$ to $<\mathrm{I} / \mathrm{I} 00)$ \\
\hline
\end{tabular}


atypia approximately 3 months after the last dose. In most patients $(81.8 \%)$, the endometrium remained atrophic after 12-month $60 \mathrm{mg}$ ospemifene treatment. A mean $0.8 \mathrm{~mm}$ increase in endometrial thickness was observed in $60 \mathrm{mg}$ ospemifene-treated patients vs $0.06 \mathrm{~mm}$ with placebo, with no significant differences in the percentage of patients with $\geq 5 \mathrm{~mm}$ endometrial thickness between both arms ( $6.6 \%$ for ospemifene vs $2.62 \%$ for placebo; $P=0.2$ ). Additionally, there was no increase in vaginal bleeding incidence in the $60 \mathrm{mg}$ ospemifene-treated women $(0.9 \%)$ vs placebo $(0.5 \%)(P=0.4){ }^{77}$

Regarding the breast, clinical evidence also suggests a beneficial effect of $60 \mathrm{mg}$ ospemifene as it demonstrated a $2 \%$ annual reduction in the incidence of abnormal findings (although not clinically significant) at breast palpation, and a $4 \%$ annual reduction in mammography findings. However, in the placebo group, abnormal mammography results increased by $2.2 \%$ after 1 year. AR rate in the breast was similar between $60 \mathrm{mg}$ ospemifene and placebo $(2.5 \%$ vs $2.2 \%$, respectively).

\section{Cardiovascular safety and venous thromboembolism}

The results from all Phase II/III studies showed no $60 \mathrm{mg}$ ospemifene-associated cardiovascular event risk increase. However, the annual incidence of venous thromboembolism (VTE) episodes with $60 \mathrm{mg}$ ospemifene was 1.6\%. Although the incidence was lower than with other SERMs such as bazedoxifene $(4.2 \%)^{78}$ or raloxifene $(4.7 \%),{ }^{79}$ it suggests that VTE risk cannot be ruled out.

\section{Safety in bone, coagulation, lipid, and weight markers} Additionally, $60 \mathrm{mg}$ ospemifene did not show any significant effect on most bone turnover markers in women without osteoporosis, ${ }^{49}$ or on coagulation, ${ }^{65-69}$ lipid $^{65-69}$ or weight $^{65-69}$ parameters.

\section{Discussion and conclusion}

Menopause is a natural aging process and represents a challenge for women's preventive healthcare and quality of life, ${ }^{80}$ even though most women do not have such perception. ${ }^{25,81}$ With lifespan increase, women will live in a postmenopausal state for more than one third of their lives, experiencing symptoms related to physiological changes associated with estrogen deficiency which affect nearly all body systems chronically. ${ }^{13,80}$ However, whereas hot flashes and night sweats are the most generally recognized menopausal symptoms, ${ }^{82}$ and although $50 \%$ of menopausal women have vaginal discomfort, ${ }^{76}$ today, most women still have little awareness of VVA, they have absolutely no idea about the origin of their symptoms, ${ }^{25}$ and they even ignore this as a chronic condition requiring treatment. ${ }^{25}$ This lack of awareness is an issue of concern, especially considering that these women are living a life period where these symptoms can have a greater impact on them..$^{25}$ Therefore, it should be highlighted that menopause is a significant health problem, and that its management is a challenge for the physician, who should probe into vaginal symptoms and talk with patients about what they themselves consider as an embarrassing issue, but which will really improve their quality of life, ${ }^{13}$ along with their sexual sphere and selfesteem. A greater dissemination of information on vulvar and vaginal health in postmenopausal women and on the importance of an effective treatment would contribute to a greater defense and active participation in the therapeutic decision-making process. ${ }^{82}$ This is all the more important as not all current VVA treatments are adequate for all postmenopausal women, and not all women adapt to their convenience and administration mode or respond favorably in terms of efficacy and safety.

The decrease in estrogen therapy use for menopausal symptoms after the WHI study's safety results ${ }^{9}$ opened the way for nonhormonal alternative therapies to treat VVA symptoms, given that concerns about the potential stimulating effects of estrogens are obvious with local vaginal therapy. ${ }^{81}$ The use of over-the-counter moisturizers and lubricants provides temporary relief of symptoms and is recommended in postmenopausal women with mild-tomoderate symptoms, ${ }^{21}$ but they do not treat the underlying condition.

The lack of therapeutic adherence shown by up to $44 \%$ of postmenopausal women participating in the REVIVE survey, who dropped out of at least one VVA treatment, ${ }^{81}$ is a clear warning about the limitations of current treatments. Apart from the dissatisfaction these women have in terms of safety and efficacy with over-the-counter products and medically prescribed vaginal therapies, $12 \%-20 \%$ claim they are uncomfortable to apply, interfere with sexual spontaneity, increase itching and burning sensation in the vulvar area, and produce vaginal flow. ${ }^{81}$ Many other women do not feel comfortable with the treatment's vaginal insertion ${ }^{25}$ and identify the vaginal route as a limitation. What is more, $33 \%-43 \%$ of postmenopausal women in Europe would prefer oral treatment. ${ }^{81}$

Selectively targeted SERMs against VVA's underlying physiopathology could be an alternative to local estrogen or systemic hormone therapy in the management of VVA 
symptoms, apart from moisturizers and lubricants. However, ospemifene is the only marketed SERM which has demonstrated an estrogen agonist effect in the vaginal epithelium. It is the first nonhormonal, nonestrogenic drug indicated for moderate-to-severe VVA treatment in women not eligible for vaginal estrogen therapy, ${ }^{83}$ as outlined in its data sheet. Ospemifene treats the underlying cause of vaginal dryness and dyspareunia, regenerating vaginal cells, improving lubrication, and reducing pain during sexual intercourse. ${ }^{74-76}$ VVA symptoms improve in most women receiving ospemifene, ${ }^{72}$ and they begin to revert following the first 4 weeks. Physiological improvements endure for up to 1 year of daily ospemifene use, as well as those related to all sexual function aspects. Apart from its proven safety profile in the endometrium and the bone and cardiovascular systems, its safety in the breast makes it the first VVA oral treatment not contraindicated in women with a previous history of breast cancer who have completed treatment. ${ }^{84}$

\section{Disclosure}

Dr Santiago Palacios had a financial relationship (lecturer, member of advisory boards and/or consultant) with Pfizer, Servier, Amgen, MSD, Preglem, Gynea, Sandoz, Procare Health, Bayer, MSD, Serelys and Shionogi. Dr Santiago Palacios has been a symposium speaker or advisory board member and has received research grants and/or consulting fees Servier, Pfizer, GSK, Abbott, Ferrer, Bioiberica, Shionogi, Amgen, Novo Nordisk, Teva, Bayer Healthcare, Serelys and Gedeon Ritcher. Dr María Jesús Cancelo has been a symposium speaker or advisory board member for Shionogi, Amgen, Grünentahal, MSD.

\section{References}

1. Mac Bride MB, Rhodes DJ, Shuster LT. Vulvovaginal atrophy. Mayo Clin Proc. 2010;85(1):87-94.

2. Portman DJ, Gass ML; Vulvovaginal Atrophy Terminology Consensus Conference Panel. Genitourinary syndrome of menopause: new terminology for vulvovaginal atrophy from the International Society for the Study of Women's Sexual Health and The North American Menopause Society. Climacteric. 2014;17(5):557-563.

3. Davila GW, Singh A, Karapanagiotou I, et al. Are women with urogenital atrophy symptomatic? Am J Obstet Gynecol. 2003;188(2): 382-388.

4. Stone SC, Mickal A, Rye PH. Postmenopausal symptomatology, maturation index, and plasma estrogen levels. Obstet Gynecol. 1975;45(6): 625-627.

5. Gass ML, Cochrane BB, Larson JC, et al. Patterns and predictors of sexual activity among women in the Hormone Therapy trials of the Women's Health Initiative. Menopause. 2011;18(11):1160-1171.

6. Huang AJ, Moore EE, Boyko EJ, et al. Vaginal symptoms in postmenopausal women: self-reported severity, natural history, and risk factors. Menopause. 2010;17(1):121-126.
7. Palacios S. Advances in hormone replacement therapy: making the menopause manageable. BMC Womens Health. 2008;8:22.

8. Hsia J, Simon JA, Lin F, et al. Peripheral arterial disease in randomized trial of estrogen with progestin in women with coronary heart disease: the Heart and Estrogen/Progestin Replacement Study. Circulation. 2000;102(18):2228-2232.

9. Rossouw JE, Anderson GL, Prentice RL, et al. Risks and benefits of estrogen plus progestin in healthy postmenopausal women: principal results From the Women's Health Initiative randomized controlled trial. JAMA. 2002;288(3):321-333.

10. Roberts H. Managing the menopause. BMJ. 2007;334(7596): 736-741.

11. Notelovitz M, Lenihan JP, McDermott M, Kerber IJ, Nanavati N, Arce J. Initial 17 beta-estradiol dose for treating vasomotor symptoms. Obstet Gynecol. 2000;95(5):726-731.

12. Utian WH, Shoupe D, Bachmann G, Pinkerton JV, Pickar JH. Relief of vasomotor symptoms and vaginal atrophy with lower doses of conjugated equine estrogens and medroxyprogesterone acetate. Fertil Steril. 2001;75(6):1065-1079.

13. Sturdee DW, Panay N; International Menopause Society Writing Group. Recommendations for the management of postmenopausal vaginal atrophy. Climacteric. 2010;13(6):509-522.

14. Simon JA. Identifying and treating sexual dysfunction in postmenopausal women: the role of estrogen. J Womens Health (Larchmt). 2011; 20(10): 1453-1465.

15. Suckling J, Lethaby A, Kennedy R. Local oestrogen for vaginal atrophy in postmenopausal women. Cochrane Database Syst Rev. 2006;(4): CD001500.

16. Rees M, Perez-Lopez FR, Ceasu I, et al. EMAS clinical guide: lowdose vaginal estrogens for postmenopausal vaginal atrophy. Maturitas. 2012;73(2):171-174.

17. Al-Baghdadi O, Ewies AA. Topical estrogen therapy in the management of postmenopausal vaginal atrophy: an up-to-date overview. Climacteric. 2009;12(2):91-105.

18. Santen RJ, Allred DC, Ardoin SP, et al. Postmenopausal hormone therapy: an Endocrine Society scientific statement. J Clin Endocrinol Metab. 2010;95(7 Suppl 1):s1-s66.

19. Labrie F, Cusan L, Gomez JL, et al. Effect of one-week treatment with vaginal estrogen preparations on serum estrogen levels in postmenopausal women. Menopause. 2009;16(1):30-36.

20. Eugster-Hausmann M, Waitzinger J, Lehnick D. Minimized estradiol absorption with ultra-low-dose 10 microg 17 beta-estradiol vaginal tablets. Climacteric. 2010;13(3):219-227.

21. No authors listed. Management of symptomatic vulvovaginal atrophy: 2013 position statement of The North American Menopause Society. Menopause. 2013;20(9):888-902.

22. Sanchez-Borrego R, Manubens M, Navarro MC, et al. Position of the Spanish Menopause Society regarding vaginal health care in postmenopausal women. Maturitas. 2014;78(2):146-150.

23. Cano A, Estevez J, Usandizaga R, et al. The therapeutic effect of a new ultra low concentration estriol gel formulation $(0.005 \%$ estriol vaginal gel) on symptoms and signs of postmenopausal vaginal atrophy: results from a pivotal phase III study. Menopause. 2012;19(10):1130-1139.

24. Santoro N, Komi J. Prevalence and impact of vaginal symptoms among postmenopausal women. $J$ Sex Med. 2009;6(8):2133-2142.

25. Nappi RE, Kokot-Kierepa M. Vaginal Health: Insights, Views \& Attitudes (VIVA) - results from an international survey. Climacteric. 2012;15(1): 36-44.

26. Sievert LL, Saliba M, Reher D, et al. The medical management of menopause: a four-country comparison care in urban areas. Maturitas. 2008; 59(1):7-21.

27. Sanchez-Borrego R, Mendoza N, Beltran E, et al. Position of the Spanish Menopause Society regarding the management of menopausal symptoms in breast cancer patients. Maturitas. 2013;75(3):294-300.

28. Biglia N, Peano E, Sgandurra P, et al. Low-dose vaginal estrogens or vaginal moisturizer in breast cancer survivors with urogenital atrophy: a preliminary study. Gynecol Endocrinol. 2010;26(6):404-412. 
29. Palacios S. Managing urogenital atrophy. Maturitas. 2009;63(4): 315-318.

30. Hainer BL, Gibson MV. Vaginitis. Am Fam Physician. 2011;83(7): $807-815$.

31. Chen J, Geng L, Song X, Li H, Giordan N, Liao Q. Evaluation of the efficacy and safety of hyaluronic acid vaginal gel to ease vaginal dryness: a multicenter, randomized, controlled, open-label, parallel-group, clinical trial. J Sex Med. 2013;10(6):1575-1584.

32. Tedeschi C, Benvenuti C; Research Group EG. Comparison of vaginal gel isoflavones versus no topical treatment in vaginal dystrophy: results of a preliminary prospective study. Gynecol Endocrinol. 2012;28(8):652-654.

33. Ghazanfarpour M, Latifnejad Roudsari R, Treglia G, Sadeghi R. Topical administration of isoflavones for treatment of vaginal symptoms in postmenopausal women: a systematic review of randomised controlled trials. J Obstet Gynaecol. 2015;35(8):783-787.

34. Pinkerton JV, Stanczyk FZ. Clinical effects of selective estrogen receptor modulators on vulvar and vaginal atrophy. Menopause. 2014; 21(3):309-319.

35. Berrodin TJ, Chang KC, Komm BS, Freedman LP, Nagpal S. Differential biochemical and cellular actions of Premarin estrogens: distinct pharmacology of bazedoxifene-conjugated estrogens combination. Mol Endocrinol. 2009;23(1):74-85.

36. Katzenellenbogen BS, Katzenellenbogen JA. Biomedicine. Defining the "S" in SERMs. Science. 2002;295(5564):2380-2381.

37. Kharode Y, Bodine PV, Miller CP, Lyttle CR, Komm BS. The pairing of a selective estrogen receptor modulator, bazedoxifene, with conjugated estrogens as a new paradigm for the treatment of menopausal symptoms and osteoporosis prevention. Endocrinology. 2008;149(12):6084-6091.

38. Komm BS. A new approach to menopausal therapy: the tissue selective estrogen complex. Reprod Sci. 2008;15(10):984-992.

39. Martinkovich S, Shah D, Planey SL, Arnott JA. Selective estrogen receptor modulators: tissue specificity and clinical utility. Clin Interv Aging. 2014;9:1437-1452.

40. Goldstein SR, Siddhanti S, Ciaccia AV, Plouffe L Jr. A pharmacological review of selective oestrogen receptor modulators. Hum Reprod Update. 2000;6(3):212-224.

41. Tee MK, Rogatsky I, Tzagarakis-Foster C, et al. Estradiol and selective estrogen receptor modulators differentially regulate target genes with estrogen receptors alpha and beta. Mol Biol Cell. 2004;15(3): $1262-1272$.

42. Taylor HS. Designing the ideal selective estrogen receptor modulatoran achievable goal? Menopause. 2009;16(3):609-615.

43. Shanle EK, Xu W. Selectively targeting estrogen receptors for cancer treatment. Adv Drug Deliv Rev. 2010;62(13):1265-1276.

44. Maximov PY, Lee TM, Jordan VC. The discovery and development of selective estrogen receptor modulators (SERMs) for clinical practice. Curr Clin Pharmacol. 2013;8(2):135-155.

45. Davies GC, Huster WJ, Lu Y, Plouffe L Jr, Lakshmanan M. Adverse events reported by postmenopausal women in controlled trials with raloxifene. Obstet Gynecol. 1999;93(4):558-565.

46. European Medicines Agency. Fareston Summary of Product Characteristics. EMA; 2014. Available from: http://www.ema.europa. eu/docs/en_GB/document_library/EPAR_-_Product_Information/ human/000091/WC500020689.pdf. Accessed June 1, 2016.

47. European Medicines Agency. Conbriza Summary of Product Characteristics. EMA; 2011. Available from: http://www.ema.europa. eu/docs/en_GB/document_library/EPAR_-_Product_Information/ human/000913/WC500033577.pdf. Accessed June 1, 2016.

48. Delmanto A, Nahas-Neto J, Nahas EA, de Oliveira ML, Fernandes CE, Traiman P. Effect of raloxifene on the vaginal epithelium of postmenopausal women. Eur J Obstet Gynecol Reprod Biol. 2008;139(2):187-192.

49. Komi J, Lankinen KS, DeGregorio M, et al. Effects of ospemifene and raloxifene on biochemical markers of bone turnover in postmenopausal women. J Bone Miner Metab. 2006;24(4):314-318.

50. Marttunen MB, Cacciatore B, Hietanen P, et al. Prospective study on gynaecological effects of two antioestrogens tamoxifen and toremifene in postmenopausal women. Br J Cancer. 2001;84(7):897-902.
51. Vardy MD, Lindsay R, Scotti RJ, et al. Short-term urogenital effects of raloxifene, tamoxifen, and estrogen. Am J Obstet Gynecol. 2003;189(1): $81-88$.

52. Varras M, Polyzos D, Akrivis CH. Effects of tamoxifen on the human female genital tract: review of the literature. Eur J Gynaecol Oncol. 2003;24(3-4):258-268.

53. Polin SA, Ascher SM. The effect of tamoxifen on the genital tract. Cancer Imaging. 2008;8:135-145.

54. Parsons A, Merritt D, Rosen A, et al. Effect of raloxifene on the response to conjugated estrogen vaginal cream or nonhormonal moisturizers in postmenopausal vaginal atrophy. Obstet Gynecol. 2003;101(2):346-352.

55. Pinkerton JV, Shifren JL, La Valleur J, Rosen A, Roesinger M, Siddhanti S. Influence of raloxifene on the efficacy of an estradiolreleasing ring for treating vaginal atrophy in postmenopausal women. Menopause. 2003;10(1):45-52.

56. Kagan R. The tissue selective estrogen complex: a novel approach to the treatment of menopausal symptoms. $J$ Womens Health (Larchmt). 2012;21(9):975-981.

57. Lobo RA, Pinkerton JV, Gass ML, et al. Evaluation of bazedoxifene/ conjugated estrogens for the treatment of menopausal symptoms and effects on metabolic parameters and overall safety profile. Fertil Steril. 2009;92(3):1025-1038.

58. Kagan R, Williams RS, Pan K, Mirkin S, Pickar JH. A randomized, placebo- and active-controlled trial of bazedoxifene/conjugated estrogens for treatment of moderate to severe vulvar/vaginal atrophy in postmenopausal women. Menopause. 2010;17(2):281-289.

59. Cui Y, Zong H, Yan H, Li N, Zhang Y. The efficacy and safety of ospemifene in treating dyspareunia associated with postmenopausal vulvar and vaginal atrophy: a systematic review and meta-analysis. $J$ Sex Med. 2014;11(2):487-497.

60. Voipio SK, Komi J, Kangas L, Halonen K, DeGregorio MW, Erkkola RU. Effects of ospemifene (FC-1271a) on uterine endometrium, vaginal maturation index, and hormonal status in healthy postmenopausal women. Maturitas. 2002;43(3):207-214.

61. Kangas L, Unkila M. Tissue selectivity of ospemifene: pharmacologic profile and clinical implications. Steroids. 2013;78(12-13):1273-1280.

62. Qu Q, Zheng H, Dahllund J, et al. Selective estrogenic effects of a novel triphenylethylene compound, FC1271a, on bone, cholesterol level, and reproductive tissues in intact and ovariectomized rats. Endocrinology. 2000;141(2):809-820.

63. Taras TL, Wurz GT, DeGregorio MW. In vitro and in vivo biologic effects of Ospemifene (FC-1271a) in breast cancer. J Steroid Biochem Mol Biol. 2001;77(4-5):271-279.

64. European Medicines Agency. Senshio: European Public Assessment Report. Report No: EMEA/H/C/002780/0000. London (UK): European Medicines Agency; 2014. Available from: http://www.ema.europa.eu/ docs/en_GB/document_library/EPAR_-_Public_assessment_report/ human/002780/WC500182777.pdf. Accessed June 1, 2016.

65. Bachmann GA, Komi JO; Ospemifene Study Group. Ospemifene effectively treats vulvovaginal atrophy in postmenopausal women: results from a pivotal phase 3 study. Menopause. 2010;17(3):480-486.

66. Portman D, Palacios S, Nappi RE, Mueck AO. Ospemifene, a nonoestrogen selective oestrogen receptor modulator for the treatment of vaginal dryness associated with postmenopausal vulvar and vaginal atrophy: a randomised, placebo-controlled, phase III trial. Maturitas. 2014;78(2):91-98.

67. Portman DJ, Bachmann GA, Simon JA; Ospemifene Study Group. Ospemifene, a novel selective estrogen receptor modulator for treating dyspareunia associated with postmenopausal vulvar and vaginal atrophy. Menopause. 2013;20(6):623-630.

68. Goldstein SR, Bachmann GA, Koninckx PR, et al. Ospemifene 12-month safety and efficacy in postmenopausal women with vulvar and vaginal atrophy. Climacteric. 2014;17(2):173-182.

69. Simon JA, Lin VH, Radovich C, Bachmann GA; Ospemifene Study Group. One-year long-term safety extension study of ospemifene for the treatment of vulvar and vaginal atrophy in postmenopausal women with a uterus. Menopause. 2013;20(4):418-427. 
70. Simon J, Portman D, Mabey RG Jr; Ospemifene Study Group. Longterm safety of ospemifene (52-week extension) in the treatment of vulvar and vaginal atrophy in hysterectomized postmenopausal women. Maturitas. 2014;77(3):274-281.

71. Soe LH, Wurz GT, Kao CJ, DeGregorio MW. Ospemifene for the treatment of dyspareunia associated with vulvar and vaginal atrophy: potential benefits in bone and breast. Int $J$ Womens Health. 2013;5: 605-611.

72. Nappi RE, Panay N, Bruyniks N, Castelo-Branco C, De Villiers TJ, Simon JA. The clinical relevance of the effect of ospemifene on symptoms of vulvar and vaginal atrophy. Climacteric. 2015;18(2):233-240.

73. Constantine G, Graham S, Koltun WD, Kingsberg SA. Assessment of ospemifene or lubricants on clinical signs of VVA. J Sex Med. 2014; 11(4):1033-1041.

74. Constantine G, Graham S, Portman DJ, Rosen RC, Kingsberg SA. Female sexual function improved with ospemifene in postmenopausal women with vulvar and vaginal atrophy: results of a randomized, placebo-controlled trial. Climacteric. 2015;18(2):226-232.

75. Ettinger B, Hait H, Reape KZ, Shu H. Measuring symptom relief in studies of vaginal and vulvar atrophy: the most bothersome symptom approach. Menopause. 2008;15(5):885-889.

76. Nappi RE, Palacios S. Impact of vulvovaginal atrophy on sexual health and quality of life at postmenopause. Climacteric. 2014;17(1):3-9.

77. Constantine GD, Goldstein SR, Archer DF. Endometrial safety of ospemifene: results of the phase $2 / 3$ clinical development program. Menopause. 2015;22(1):36-43.
78. Christiansen C, Chesnut CH 3rd, Adachi JD, et al. Safety of bazedoxifene in a randomized, double-blind, placebo- and active-controlled Phase 3 study of postmenopausal women with osteoporosis. BMC Musculoskelet Disord. 2010;11:130.

79. Grady D, Ettinger B, Moscarelli E, et al. Safety and adverse effects associated with raloxifene: multiple outcomes of raloxifene evaluation. Obstet Gynecol. 2004;104(4):837-844.

80. Gennari L, Merlotti D, Valleggi F, Nuti R. Ospemifene use in postmenopausal women. Expert Opin Investig Drugs. 2009;18(6):839-849.

81. Kingsberg SA, Wysocki S, Magnus L, Krychman ML. Vulvar and vaginal atrophy in postmenopausal women: findings from the REVIVE (REal Women's VIews of Treatment Options for Menopausal Vaginal ChangEs) survey. J Sex Med. 2013;10(7):1790-1799.

82. Parish SJ, Nappi RE, Krychman ML, et al. Impact of vulvovaginal health on postmenopausal women: a review of surveys on symptoms of vulvovaginal atrophy. Int J Womens Health. 2013;5:437-447.

83. DeGregorio MW, Zerbe RL, Wurz GT. Ospemifene: a first-in-class, non-hormonal selective estrogen receptor modulator approved for the treatment of dyspareunia associated with vulvar and vaginal atrophy. Steroids. 2014;90:82-93.

84. European Medicines Agency. Senshio Summary of Product Characteristics. EMA; 2015. Available from: http://www.ema.europa. eu/docs/en_GB/document_library/EPAR_-_Product_Information/ human/002780/WC500182775.pdf. Accessed June 1, 2016.
International Journal of Women's Health

\section{Publish your work in this journal}

The International Journal of Women's Health is an international, peerreviewed open-access journal publishing original research, reports, editorials, reviews and commentaries on all aspects of women's healthcare including gynecology, obstetrics, and breast cancer. The manuscript management system is completely online and includes

\section{Dovepress}

a very quick and fair peer-review system, which is all easy to use. Visit http://www.dovepress.com/testimonials.php to read real quotes from published authors. 